\title{
Proper-motion studies of Milky Way starburst clusters - a new definition of starburst cluster templates
}

\author{
Andrea Stolte ${ }^{1}$ and Wolfgang Brandner ${ }^{2}$ \\ ${ }^{1}$ I. Physikalisches Institut, University of Cologne, Zülpicher Str. 77, 50937 Köln, Germany \\ email: astolte@ph1.uni-koeln.de \\ ${ }^{2}$ Max-Planck-Institut für Astronomie, Königstuhl 17, 69117 Heidelberg, Germany \\ email: brandner@mpia.de
}

\begin{abstract}
Starburst clusters in the Milky Way have the advantage that individual stars down to subsolar masses can be resolved. Thus far, field contamination along the line of sight towards the Galactic Centre and spiral arms was the limiting factor in deriving an unbiased census of the stellar population in Milky Way starbursts and, hence, the spatial extent and initial mass function in starburst clusters. As the next generation of telescopes with higher sensitivity and spatial resolution are being developed, these resolved clusters become increasingly important as templates for young, massive extragalactic systems, which will be resolved at the high-mass end of the stellar mass function. With the aim to obtain a uniform characterisation of starburst cluster properties in the Milky Way, we have initiated a proper-motion membership survey. This technique became feasible for clusters out to distances of $8 \mathrm{kpc}$ with diffraction-limited imaging using adaptive optics from the ground and with Hubble Space Telescope from space.
\end{abstract}

Keywords. techniques: high angular resolution, astrometry, stars: formation, stars: pre-mainsequence, stars: luminosity function, mass function, open clusters and associations: general, galaxies: star clusters

\section{Introduction}

Starburst clusters, here defined as young, massive clusters with a total stellar mass in excess of $10^{4} \mathrm{M}_{\odot}$, form one of the major building blocks of galaxies. In extragalactic systems, such as the merging Antennae galaxies or galaxies with rich cluster populations such as M51, several hundreds of young, massive clusters with ages $<100$ Myr are observed (e.g., Whitmore \& Schweizer 1995; Lee et al. 2005). At the low star-formation rate in the Milky Way today, starburst clusters are rare. Yet, as they contain the densest stellar environments outside the Galactic Centre, their stellar populations can only be resolved in the Galaxy in their entirety, and outside the immediate cluster cores in the Magellanic Clouds. Despite our proximity to the nearest starburst clusters at distances of 4 to $8 \mathrm{kpc}$ from the Sun, several cluster characteristics are not well quantified. Among these are the full cluster extent, the amount of mass segregation, the core radii, and, most importantly, the stellar initial mass function (IMF). On theoretical grounds, one might expect that starburst cluster environments lead to an overproduction of highmass stars as a consequence of increased temperatures, densities, accretion rates and possibly merging pre-stellar cores in the dense cluster centres. In practice, however, it is difficult to distinguish the effects of a flattened stellar initial mass function (MF) from the flattening induced by dynamical mass segregation. Hence, while present-day MFs in the Arches and NGC 3603 cluster cores were found to be flattened with respect 

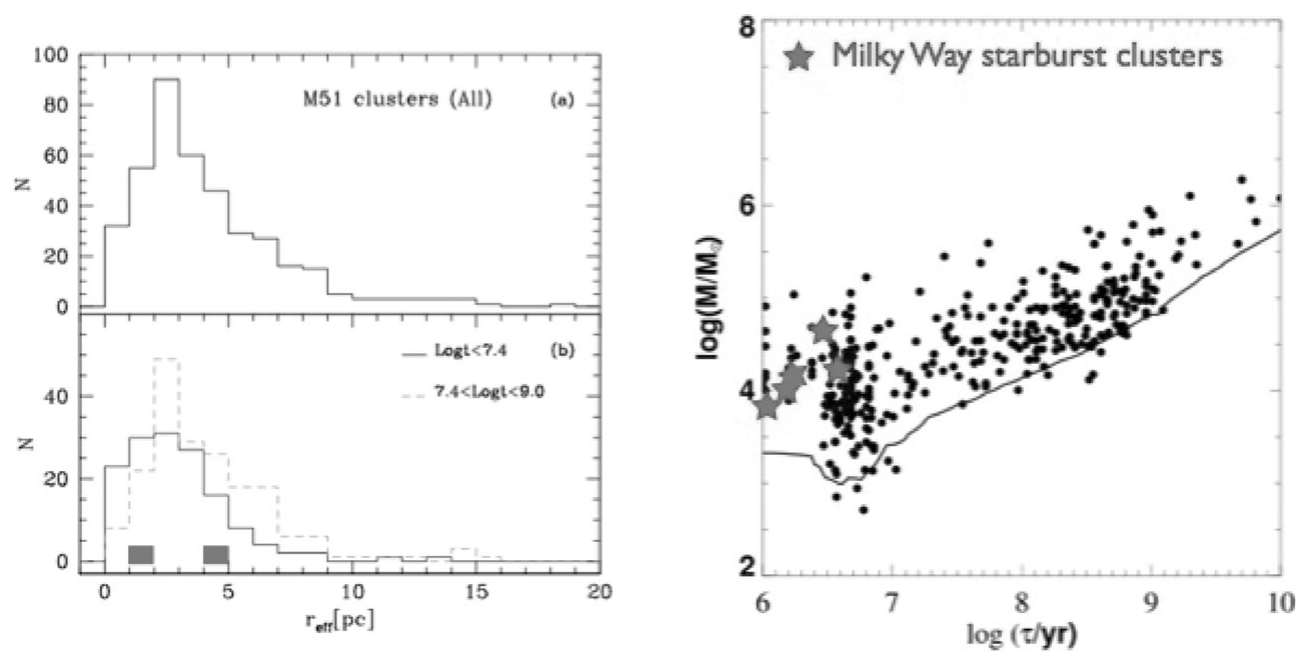

Figure 1. Comparison of Milky Way starburst cluster characteristics with young star clusters in the merging galaxy M51 (the Whirlpool galaxy). The four clusters in the survey span similar sizes as the young clusters in M51, and cover, in particular, the low-mass end of the cluster population, which will be detected with the next generation of high-resolution telescopes such as the E-ELT and the TMT. (Figures adapted from Lee et al. 2005.)

to a Salpeter IMF (Figer et al. 1999; Stolte et al. 2005, 2006; Harayama et al. 2008), conclusions on the IMF are difficult to deduce in the presence of incomplete cluster populations, unknown radial extent and rapid mass segregation (see Kim et al. 2006 for a detailed discussion). Recently, Espinoza et al. (2009) showed that incomplete samples caused by variable extinction across the field can mimic a flattening in the IMF. With different assumptions than previous studies, these authors derive a normal present-day MF for the Arches cluster. The deviating results emphasize the need to obtain complete samples and understand the possible biases in each particular data set. At the same time, a homogeneous treatment of the data would help the relative comparison between different clusters.

The fact that even the closest starburst cluster populations are not well constrained has important implications for our understanding of extragalactic star clusters. The light of unresolved extragalactic clusters is dominated by the most massive O-type stars, while the total cluster mass and number density are determined by low- and intermediatemass stars. The interpretation of the observed integrated light profile, therefore, depends crucially on assumptions regarding the true cluster extent and the stellar IMF.

\section{The Milky Way starburst cluster survey}

Local clusters can serve as calibrators for extragalactic cluster systems, if their properties are well understood. A complete census of cluster members in each local starburst is crucial to quantify mass segregation, dynamical cluster dispersal, the IMF and the survival timescale before clusters are dispersed into the field. With the aim to map each cluster population as completely as possible, we initiated a proper-motion membership survey of four Milky Way starburst clusters (the Arches, Quintuplet, NGC 3603 and Westerlund 1). Extended regions around each cluster are monitored out to at least the tidal radius $(1-2 \mathrm{pc})$, such that ejected stars and low-mass stars transported to the cluster outskirts can be detected. 

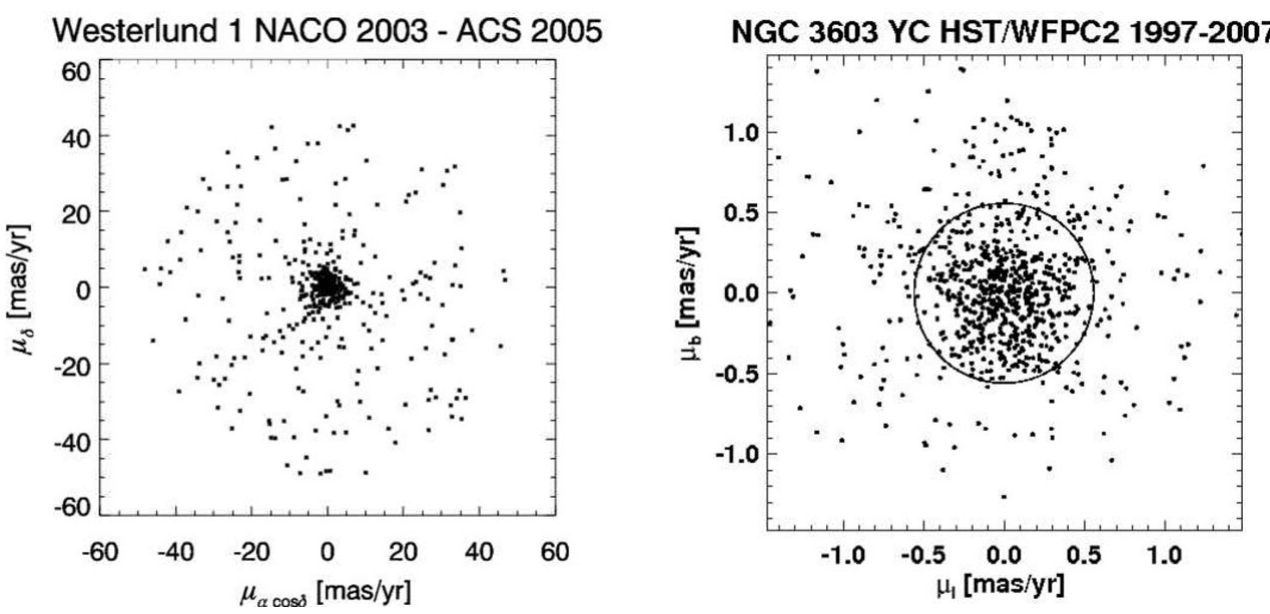

Figure 2. Proper-motion diagrams for Westerlund 1 (left) and NGC 3603 (right). The colourmagnitude diagrams in Figure 3 were derived based on a $1 \sigma$ membership-selection criterion. Field stars occupying a larger range of Galactic orbits and hence a large spread in velocities are efficiently removed from the cluster samples.

Proper-motion membership selections are used very efficiently in nearby star-forming regions (e.g., Kharchenko et al. 2009; and references therein). Recently, we were able to apply this technique for the first time to the Arches starburst cluster at a distance of $8 \mathrm{kpc}$, near the center of the Galaxy (Stolte et al. 2008). The clear distinction between field and cluster stars allows sampling the cluster stars among the dominant field population out to large radii, and hence enables determination of the cluster extent and the distribution of low-mass members in particular.

The goals of the survey are to obtain proper-motion membership out to the tidal radius in each cluster, determine the present cluster extent, and derive the unbiased stellar IMF. Furthermore, the internal cluster dynamics will constrain the tidal disruption and dynamical evolution of starburst clusters. In particular, the combination with radial velocities reveals possible deviations from virial equilibrium, which are crucial for measuring dynamical cluster masses. This is particularly important for extragalactic clusters, where the velocity dispersion and/or the mass-to-light ratio are employed to derive the total cluster mass and survival timescale, and to constrain the existence of intermediate-mass black holes at the centres of starburst clusters (e.g., Gürkan et al. 2004; Portegies Zwart et al. 2004; and references therein).

\section{The method}

With diffraction-limited imaging using adaptive optics from the ground (near-infrared) or HST/WFPC2 from space (optical), a spatial resolution of 50-80 milliarcseconds (mas) is achieved with pixel scales of 27-40 mas. In deep astrometric observations, a positional uncertainty of $\frac{1}{20}$ of a pixel is now routinely obtained, or a relative accuracy of 1-2 mas (rms). With a time baseline of only two years, this implies that cluster and field stars can be separated at the level of $30 \mathrm{~km} \mathrm{~s}^{-1}$, sufficient to distinguish the concentrated cluster population from the diverse orbits of stars along the line of sight (Figure 2).

The survey sample contains four of the best-studied and most massive young starburst clusters in the Milky Way. With the Arches and Quintuplet, two clusters represent the Galactic Centre's star-forming environment, which is most representative for the central 

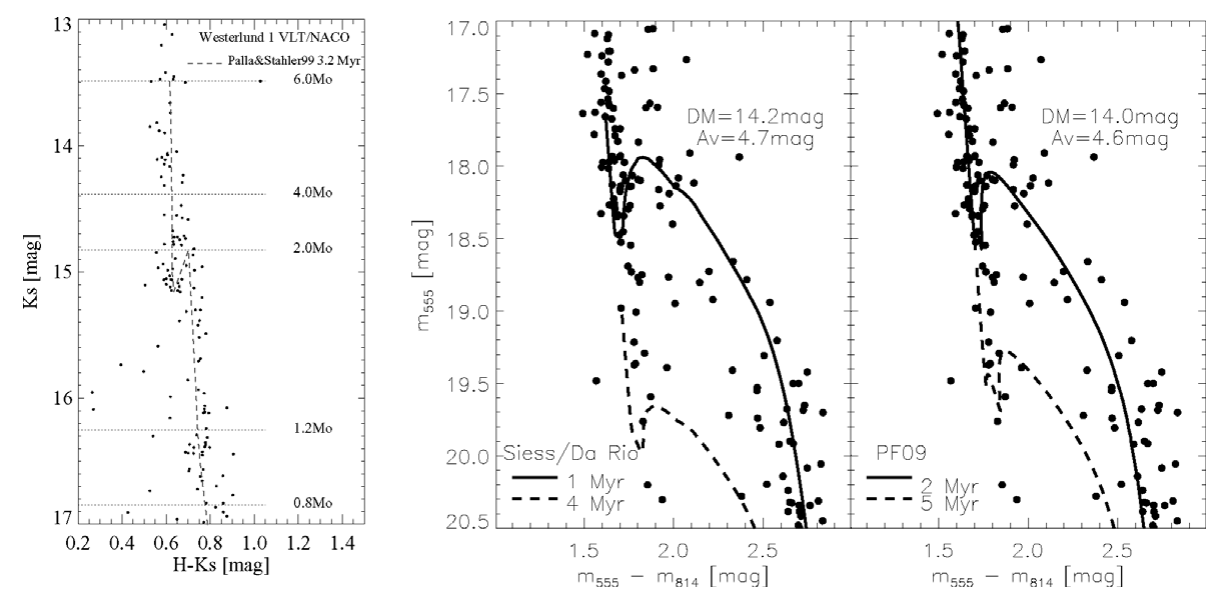

Figure 3. Colour-magnitude diagrams created from the membership selection shown in Figure 2. The membership selection in Westerlund 1 (left) allowed a significantly more accurate determination of the cluster distance and age. In NGC 3603 YC (Young Cluster), the sample selected from HST/WFPC2 imaging based on a 10-year baseline displays at least two distinctive hydrogen-burning turn-on points, indicating multiple star-formation epochs. The photometric precision and membership selection provide new constraints on pre-main-sequence evolution models. While the Siess models (left) do not reproduce the tip of the youngest hydrogen-burning turn-on point, the newest generation of simulations by the Pisa group (right; Tognelli et al., in prep.) trace this phase extremely well.

regions of galaxies such as M31 (Andromeda) or M51 (the Whirlpool). NGC 3603 YC (Young Cluster) at the heart of the giant HII region NGC 3603 and the very massive cluster Westerlund 1 (Clark et al. 2005; Brandner et al. 2008) evolve in the more quiescent environment of the spiral arms. With ages of 2 to $5 \mathrm{Myr}$, these clusters span the age range where planetary-system formation takes place. Compared to the cluster sample in the star-forming merger M51 (Figure 1), the four starburst clusters in the survey display comparable cluster sizes and occupy the low-mass range of young extragalactic clusters. Hence, despite the small number of clusters available in the Milky Way, a significant range of ages and environments is covered to study cluster evolution.

For each of the four starburst clusters in the sample, we obtained first-epoch VLT/ NAOS-CONICA (NACO) observations, which were combined with previous observations of each cluster core. In addition, several fields around the immediate core regions were observed to reach large clustercentric distances. For the extended cluster areas, a secondepoch observation will be obtained in the next few years. The astrometric precision can be optimised by deriving the optical distortion correction for NACO, which is currently being done by N. Kudryavtseva (in prep.). For NGC 3603 we obtained two epochs of HST/WFPC2 observations separated by a 10-year baseline. From these observations, the internal velocity dispersion could be measured for the first time (Rochau et al., submitted).

\section{Preliminary results}

Constraining cluster membership implies that the global cluster properties can be derived with higher accuracy. In Brandner et al. (2008), we revised the age of Westerlund 1 from $4 \pm 1$ to $3.3 \pm 0.2 \mathrm{Myr}$, and the cluster distance from $3.9 \pm 0.7$ to $3.5 \pm 0.2 \mathrm{kpc}$. In the central starburst cluster in the giant His region NGC 3603, we were able to resolve multiple star-forming events for the first time from the membership selection in the pre-main-seqeunce/main-sequence (PMS/MS) transition region (Figure 3). The main- 
sequence turn-on point stands out clearly, so that we are now in the position to constrain stellar evolution models. In addition, stars in transition from the PMS to the MS pass through the short-lived phase of the radiative-convective gap. Only starburst clusters have sufficient members to detect several sources in this important phase of stellar evolution and constrain the physics during the hydrogen-burning equilibration phase.

\section{Summary and survey outlook}

The proper-motion survey of Milky Way starburst clusters leads to accurate cluster properties such as ages, distances and cluster extent. The photometric accuracy obtained in the colour-magnitude diagrams with member selection allowed us to constrain PMS evolution models and select the model most representative of the observed PMS colours. With the second epoch of observations in the extended cluster areas, the cluster radii will be measured and a complete sample of cluster stars with individual extinction estimates will be derived, from which the cluster IMF can be deduced.

The dynamical state of a starburst cluster has severe implications for the derivation of masses and survival timescales of young, massive extragalactic clusters, where radialvelocity measurements and assumptions on the mass-to-light ratio have to be employed. To use local resolved starbursts as templates for extragalactic clusters, it is particularly critical that the astrometric precision allows us to observe the internal velocity dispersion in each cluster and its variation with radius. In comparison with cluster evolution models (Baumgardt \& Kroupa 2008), deviations from virial equilibrium can be deduced. Quantifying the dynamical state of each cluster is also crucial to understand the effects of different environments, such as the Galactic Centre or spiral arms, on cluster evolution. Recently, Pfalzner (2009) suggested on the basis of the mass density versus radius relation in young star clusters that starburst clusters evaporate slowly with time, while low-mass clusters become rapidly unbound during the gas-expulsion phase. With a uniform sample of proper-motion-selected cluster members, the mass density and half-mass radius are more accurately determined and selection biases in the more distant starburst cluster sample compard to the sample of nearby, lower-mass star-forming clusters can be quantified.

In summary, the Milky Way starburst cluster survey will provide revised distances, ages and radial extents for the four starburst clusters the Arches, Quintuplet, NGC 3603 and Westerlund 1. The dynamical state and the stellar IMF in each cluster will be derived out to the tidal radii. These results can be applied directly when the integrated-light profiles of extragalactic young, massive clusters are interpreted. Galactic starburst cluster templates become increasingly important in view of the next generation of telescopes such as the $E-E L T$ or $T M T$, with which the high-mass end $\left(M>20 \mathrm{M}_{\odot}\right)$ of the stellar population in young, massive clusters in all nearby galaxies out to distances of several Mpc will be resolved.

\section{References}

Baumgardt, H., \& Kroupa P. 2007, MNRAS, 380, 1589

Brandner, W., Clark, J. S., Stolte, A., Waters, R., Negueruela, I., \& Goodwin, S. P. 2008, A\&A, 478,137

Clark, J. S., Negueruela, I., Crowther, P. A., \& Goodwin, S. P. 2005, A\& A, 434, 949

Espinoza, P., Selman, F., \& Melnick, J. 2008, A\&A, 501, 563

Figer D. F., Kim, S. S., Morris, M., Serabyn E., Rich, R. M., \& McLean, I. S. 1999, ApJ, 525, 750

Gürkan, M. A., Freitag, M., \& Rasio, F. A. 2004, ApJ, 604, 632 
Kharchenko, N. V., Piskunov, A. E., Röser, S., Schilbach, E., Scholz, R.-D., \& Zinnecker, H. 2009, A\&A, 504, 681

Kim, S. S., Figer, D. F., Kudritzki, R. P., \& Najarro, F. 2006, ApJ (Letters), 653, 113

Harayama, Y., Eisenhauer, F., \& Martins, F. 2008, ApJ, 675, 1319

Lee M. G., Chandar, R., \& Whitmore, B. C. 2005, AJ, 310, 2128

Pfalzner, S. 2009, A\& A, 498, L37

Portegies Zwart, S. F., Baumgardt, H., Hut, P., Makino, J., \& McMillan, S. L. W. 2004, Nature, 428,724

Stolte, A., Brandner, W., Grebel, E. K., Lenzen, R., \& Lagrange, A.-M. 2005, ApJ (Letters), $628, \mathrm{~L} 113$

Stolte, A., Brandner, W., Brandl, B., \& Zinnecker, H. 2006, AJ, 132, 253

Stolte, A., Ghez, A. M., Morris, M., Lu, J. R., Brandner, W., \& Matthews, K. 2008, ApJ, 675, 1278

Whitmore, B. C. \& Schweizer, F. 1995, AJ, 109, 960 